IP/BBSR-95-5

January 22, 1995

\title{
SYMMETRIES OF THE DIMENSIONALLY REDUCED STRING EFFECTIVE ACTION
}

\author{
Jnanadeva Maharana \\ Institute of Physics, \\ Bhubaneswar 751 005, INDIA
}

\begin{abstract}
A two dimensional string effective action is obtained by dimensionally reducing the bosonic part of the ten dimensional heterotic string effective action. It is shown that this effective action, with a few restrictions on some backgrounds describes a two dimensional model which admits an infinite sequence of nonlocal conserved currents.
\end{abstract}


The string theory is endowed with a very rich symmetry structure. Recently, the study of target space symmetries such as global $O(d, d)$ transformations including T-duality and S-duality, have attracted considerable attention [1-8]. The invariance of the string effective action under $O(d, d)$ symmetry, for the time dependent backgrounds corresponding to string cosmological scenario, was shown in ref [2] and subsequently the $O(d, d)$ transformation was applied to generate new cosmological backgrounds [3,4] satisfying the equations of motion. In a more general setting, when the backgrounds are independent of $d$ 'internal' cordinates which are toroidally compactified, the dimensional reduction technique can be employed to derive the effective action as was carried out by Schwarz and the author [6]. It was shown that the reduced action, thus derived can be cast in a manifestly $O(d, d)$ invariant form. The effective action in four spacetime dimensions has further interesting features. The field strength of the antisymmetric tensor is dual to the axion when $D=4$, D being the number of spacetime dimensions. Then, the dilaton and the axion can be combined to define a complex field. The equations of motion associated with the four dimensional action are invariant under an $S L(2, Z)$ transformation of this complex field when the appropriate transformation rules are defined for other background fields as well. However, the effective action is not invariant under this $S L(2, Z)$ transformation, although the equations of motion are. Thus the four dimensional theory, obtained by the dimensional reduction, is not only invariant under global $O(d, d)$ transformation but also is invariant under $S L(2, Z)$ transformation. We may mention in passing that whereas T-duality is a subgroup of the $O(d, d)$ transformation the S-duality is that of the $S L(2, Z)$ alluded to above.

It has been observed [9-11] recently that the three dimensional string effective action, dimensionally reduced from the ten dimensional heterotic string effective action, possesses even a larger symmetry. It has been pointed out by Sen [9] that when the ten dimensional 
theory is compactified on a seven dimensional torus the resulting target space duality group is $O(7,23 ; Z)$ as is expected. The target space duality group and the strong-weak coupling duality transformations combine into the group $O(8,28 ; Z)$. Thus we see that the four dimensional theory has $O(d, d)$ and $S L(2, Z)$ symmetry and the three dimensional theory has a larger symmetry group which incorporates both these symmetries. Recently, it has been demonstrated that if the metric, antisymmetric tensor field and the dilaton of a four dimensional effective action depend on two of the four spacetime coordinated then the resulting theory has an enlarged symmetry. Bakas has shown that the dimensionally reduced theory has a symmetry which is infinitesimally identified with the $O(2,2)$ current algebra [12]. The integrable property arises due to the presence of two commuting Killing vectors. Furthermore there is, again, an intimate relation between the S-duality and T-duality.

It is well known that two dimensional theories have several novel features and possess rich symmetry structure. One of the important attributes of a large class of two dimensional models is the integrability property and several of these models admit infinite sequence of nonlocal conserved currents in the classical analysis. These conservation laws might not survive when one considers quantum theory; however, the classical theories have attracted considerable attention in the past.

The purpose of this note is to consider an string effective action in two spacetime dimensions. For sake of definiteness we consider the ten dimensional tree level effective action of the heterotic string with graviton, antisymmetric tensor and the dilaton as the background field. This action is dimensionally reduced to two dimensions following the procedure adopted by us [6]. It is expected that the two dimensional reduced action will have interesting symmetry properties. Indeed, the theory is endowed with an infinite number of nonlocal conserved charges. This is our main result. The rest of the paper is organised 
as follows: First we recall the essential results of Maharana and Schwarz [6] to derive the two dimensional effective action. Then, the coset reconstruction of the effective action is discussed. It is essential to go over to this formulation to demonstrate the existence of nonlocal conserved currents ( NCC ). Then we explicitly construct these currents and show the conservation laws following appropriate techniques.

Let us recall how one derives the string effective action. If we consider the evolution of the string in the background of its massless excitations and require that the first quantised world sheet action respects conformal invariance, then the $\beta$-functions associated with the backgrounds must vanish. In other words, these conditions turn out to be the equations of motion to be satisfied by the backgrounds to ensure conformal invariance of the theory. The tree level string effective action, involving only the massless excitations, can be constructed in such a way that corresponding equations of motion of this effective action exactly coincide with the vanishing of the $\beta$-functions.

Let us consider the bosonic part of the tree level effective action for the heterotic string where the set of massless background fields are graviton, antisymmetric tensor and the dilaton. In what follows, we recapitulate the results of ref.6. The effective action in $\hat{D}=D+d$ dimensions $(\hat{D}=10$ for the present case $)$ is,

$$
\hat{S}=\int d^{\hat{D}} x \sqrt{-\hat{g}} e^{-\hat{\phi}}\left[\hat{R}(\hat{g})+\hat{g}^{\hat{\mu} \hat{\nu}} \partial_{\hat{\mu}} \hat{\phi} \partial_{\hat{\nu}} \hat{\phi}-\frac{1}{12} \hat{H}_{\hat{\mu} \hat{\nu} \hat{\rho}} \hat{H}^{\hat{\mu} \hat{\nu} \hat{\rho}}\right] .
$$

$\hat{H}$ is the field strength of antisymmetric tensor and $\hat{\phi}$ is the dilaton. Here we have set all the nonabelian gauge field backgrounds to zero. We consider the theory in a spacetime $M \times K$, where $M$ is $D$ dimensional space and the coordinates on $M$ are denoted by $x^{\mu}$ whereas $K$ has $d$ dimensions and $y^{\alpha}$ are the coordinates of this submanifold. When the backgrounds are independent of the 'internal' coordinates $y^{\alpha}, \alpha=1,2 . . d$ and the internal space is taken 
to be torus, the metric $\hat{g}_{\hat{\mu} \hat{\nu}}$ can be decomposed as

$$
\hat{g}_{\hat{\mu} \hat{\nu}}=\left(\begin{array}{cc}
g_{\mu \nu}+A_{\mu}^{(1) \gamma} A_{\nu \gamma}^{(1)} & A_{\mu \beta}^{(1)} \\
A_{\nu \alpha}^{(1)} & G_{\alpha \beta}
\end{array}\right),
$$

where $G_{\alpha \beta}$ is the internal metric and $g_{\mu \nu}$, the $D$-dimensional space-time metric, depend on the coordinates $x^{\mu}$. The dimensionally reduced action is,

$$
\begin{aligned}
S & =\int d^{D} x \sqrt{-g} e^{-\phi}\left\{R+g^{\mu \nu} \partial_{\mu} \phi \partial_{\nu} \phi-\frac{1}{12} H_{\mu \nu \rho} H^{\mu \nu \rho}\right. \\
& \left.+\frac{1}{8} \operatorname{tr}\left(\partial_{\mu} M^{-1} \partial^{\mu} M\right)-\frac{1}{4} \mathcal{F}_{\mu \nu}^{i}\left(M^{-1}\right)_{i j} \mathcal{F}^{\mu \nu j}\right\} .
\end{aligned}
$$

Here $\phi=\hat{\phi}-\frac{1}{2} \log \operatorname{det} G$ is the shifted dilaton.

$$
H_{\mu \nu \rho}=\partial_{\mu} B_{\nu \rho}-\frac{1}{2} \mathcal{A}_{\mu}^{i} \eta_{i j} \mathcal{F}_{\nu \rho}^{j}+(\text { cyc. perms. })
$$

$\mathcal{F}_{\mu \nu}^{i}$ is the $2 d$-component vector of field strengths

$$
\mathcal{F}_{\mu \nu}^{i}=\left(\begin{array}{c}
F_{\mu \nu}^{(1) \alpha} \\
F_{\mu \nu \alpha}^{(2)}
\end{array}\right)=\partial_{\mu} \mathcal{A}_{\nu}^{i}-\partial_{\nu} \mathcal{A}_{\mu}^{i}
$$

$A_{\mu \alpha}^{(2)}=\hat{B}_{\mu \alpha}+B_{\alpha \beta} A_{\mu}^{(1) \beta}$ (recall $\left.B_{\alpha \beta}=\hat{B}_{\alpha \beta}\right)$, and the $2 d \times 2 d$ matrices $M$ and $\eta$ are defined as

$$
M=\left(\begin{array}{cc}
G^{-1} & -G^{-1} B \\
B G^{-1} & G-B G^{-1} B
\end{array}\right), \quad \eta=\left(\begin{array}{cc}
0 & 1 \\
1 & 0
\end{array}\right)
$$

The action (3) is invariant under a global $O(d, d)$ transformation,

$$
M \rightarrow \Omega^{T} M \Omega, \quad \Omega \eta \Omega^{T}=\eta, \quad \mathcal{A}_{\mu}^{i} \rightarrow \Omega^{i}{ }_{j} \mathcal{A}_{\mu}^{j}, \quad \text { where } \quad \Omega \in O(d, d) .
$$


and the shifted dilaton, $\phi$, remains invariant under the $O(d, d)$ transformations. We mention in passing that $M \rightarrow M^{-1}$ under the duality transformation which is a generalisation of $R \rightarrow \frac{1}{R}$ duality. Note that $M \in O(d, d)$ also and $M^{T} \eta M=\eta$. The background equations of motion can be derived from the reduced action. The classical solutions of the string effective action correspond to different string vacua and are given by solutions for $M, \mathcal{F}$ and $\phi$. Thus, when one obtains a set of backgrounds satisfying the equations of motion they correspond to a vacuum configurations of the string theory. One can generate new background configurations by implementing suitable $O(d, d)$ transformations on a known solution.

Let us consider the effective action in two spacetime dimensions. We note that the term corresponding to the field strength of the antisymmetric tensor field, $B_{\mu \nu}$, does not contribute in $1+1$ dimensions. We assume that the dilaton $\phi=$ constant; this assumption is made to simplify the calculations. One can redefine the action suitably for nonconstant dilaton as has been adopted by Bakas. Next, we set the abelian field strength to zero from now on. Then the action given by (3) takes the form

$$
S=\int d^{2} x \sqrt{-g}\left\{R+\frac{1}{8} \operatorname{tr}\left(\partial_{\mu} M^{-1} \partial^{\mu} M\right)\right\} .
$$

We note that the kinetic energy term for $\phi$ does not appear in the above equation. Since we are considering two dimensional spacetime, we can choose the spacetime metric $g_{\mu \nu}=$ $e^{\alpha(x, t)} \eta_{\mu \nu}$. Here $\eta_{\mu \nu}$ is the flat diagonal spacetime metric $=\operatorname{diag}(-1,1)$ ( not to be confused with the $O(d, d)$ metric ). Since the Einstein term of the action in two dimensions is a topological term it does not contribute to the equations of motion. Thus the equations of motion associated with the matrix $\mathrm{M}$ is of primary importance to us. The present form of the action is not the most suitable one to derive the infinite set of NCC. Thus we rewrite the 
above action in a slightly different form following the approach of Maharana and Schwarz $[6]$.

The matrix $\mathrm{M}$ introduced in equation (6) can be expressed as

$$
M_{i j}=\left(V^{T} V\right)_{i j}=\delta^{A B} V_{A i} V_{B j}
$$

The matrix $\mathrm{V}$ that produces desired M-matrix is

$$
V=\left(\begin{array}{cc}
E^{-1} & -E^{-1} B \\
0 & E
\end{array}\right)
$$

where $\mathrm{E}$ is a $d \times d$ vielbein satisfying $E^{T} E=G$. Moreover, $\mathrm{V}$ is also an $O(d, d)$ matrix since $V^{T} \eta V=\eta$. We would like to construct an action which is manifestly invariant under the global $O(d, d)$ symmetry and local $O(d) \otimes O(d)$ symmetry transformation. The desired Lagrangian density is

$$
\mathcal{L}=\frac{1}{4} \eta^{i j} \eta^{A B}\left(D_{\mu} V\right)_{A i}\left(D^{\mu} V\right)_{B j}
$$

Here $\mathrm{V}$ is an arbitrary $O(d, d)$ matrix and not of the special form given by eq.(9). Since the $O(d, d)$ metric $\eta$ is off-diagonal form, it is not very convenient to handle the covariant derivatives appearing in the Lagrangian above. We can easily go over to the $O(d, d)$ metric which has $d$ diagonal +1 entries $d$ diagonal -1 entries; denoted by $\sigma$. The transformation matrix , $\rho$ that takes us from $\eta$ basis to the $\sigma$ basis is given by

$$
\rho=\frac{1}{\sqrt{2}}\left(\begin{array}{cc}
1 & -1 \\
1 & 1
\end{array}\right), \text { and }, \sigma=\left(\begin{array}{cc}
1 & 0 \\
0 & -1
\end{array}\right)
$$

Now the matrix $\mathrm{V}$ gets rotated to $W=\rho^{T} V \rho$. Recall that $V^{T} \eta V=\eta$ since $\mathrm{V}$ belongs to $O(d, d)$, the analogous condition satisfied by $\mathrm{W}$ is $W^{T} \sigma W=\sigma$. The covariant derivative 
acting on $\mathrm{W}$ fields is defined to be

$$
\left(D_{\mu} W\right)_{A i}=\partial_{\mu} W_{A i}+\omega_{\mu A B} \sigma^{B C} W_{C i}
$$

where the $O(d) \otimes O(d)$ gauge fields $\omega_{\mu}$ are given by

$$
\omega_{\mu}=\left(\begin{array}{cc}
\omega_{\mu}^{(1)} & 0 \\
0 & \omega_{\mu}^{(2)}
\end{array}\right)
$$

The Lagrangian density, expressed in terms of the $\mathrm{W}$ fields, takes the form

$$
\mathcal{L}=\frac{1}{4} \sigma^{i j} \sigma^{A B}\left(D_{\mu} W\right)_{A i}\left(D^{\mu} W\right)_{B j}
$$

The gauge fields are antisymmetric in their internal indices. Notice that the kinetic energy term for the gauge field does not appear in the Lagrangian and therefore, their equations of motion are merely constraint equations. Thus the gauge fields can be expressed in terms of $W_{A i}$ as

$$
\omega_{\mu A B}=\frac{1}{2}\left(W_{A i} \partial_{\mu} W_{B}^{i}-\partial_{\mu} W_{A i} W_{B}^{i}\right)
$$

Now, substituting the expression for the gauge fields in the Lagrangian and using the specific form of $\mathrm{V}$ matrix in that Lagrangian one shows that the corresponding action is equal to $\frac{1}{8} \operatorname{tr}\left(\partial_{\mu} M^{-1} \partial^{\mu} M\right)$. This proves that the moduli $G$ and $B$ parametrise an $\frac{O(d, d)}{O(d) \otimes O(d)} \operatorname{coset}$ $[6,13]$.

The equations of motion associated with the fields $W_{A i}$ are

$$
\left(D^{\mu} D_{\mu}\right)_{A B} W_{C}^{i} \sigma^{B C}-W_{C}^{k} \sigma^{C D} W_{D}^{i}\left(D^{\mu} D_{\mu}\right)_{A B} \sigma^{B E} W_{E k}=0
$$


As is evident $i, j, k .$. are the $O(d, d)$ index and $A, B, C .$. are the $O(d) \otimes O(d)$ indices. Some care must be exercised in deriving the equations of motion since $\mathrm{W}$ is a matrix satisfying the constraint $W^{T} \sigma W=\sigma$. Therefore, the equations of motion for the $\mathrm{W}$ fields are not just the free field equations.

We proceed to define an $O(d, d)$ gauge potential which will play a crucial role in deriving the set of conserved currents. Define

$$
\mathcal{A}_{\mu i j}=\frac{1}{2}\left(W_{A i} D_{\mu A B} W_{B j}-W_{B j} D_{\mu A B} W_{A i}\right)
$$

The covariant derivative appearing here is the same as the one defined in eq.(13). We define another covariant derivative in terms of the new gauge potential $\mathcal{A}_{\mu}$ as

$$
\mathcal{D}_{\mu}=\partial_{\mu}+\mathcal{A}_{\mu}
$$

There are two important properties satisfied by $\mathcal{A}_{\mu}$

$$
\partial^{\mu} \mathcal{A}_{\mu}=0
$$

is satisfied onshell; that is when the fields satisfy equations of motion. Furthermore, the curvature associated with $\mathcal{A}_{\mu}$ vanishes:

$$
\left[\mathcal{D}_{\mu}, \mathcal{D}_{\nu}\right]=0
$$

Now we are in a position to demonstrate the existence of the nonlocal conserved currents [14-16]. The proof is by induction. Let us assume that the $n^{\text {th }}$ conserved current, $J_{\mu}^{(n)}$, exists. Thus the current ( since we are considering two dimensional spacetime) can be expressed as 


$$
J_{\mu}^{(n)}=\epsilon_{\mu \nu} \partial^{\nu} \chi^{(n)}
$$

and the current is conserved by construction. Notice that $J^{(n)}(x, t)$ and $\chi^{(n)}(x, t)$ carry $O(d, d)$ indices $i j$ and are matrix valued objects; we suppress the indices here and everywhere. The next level current $J_{\mu}^{(n+1)}$ is constructed as follows:

$$
J_{\mu}^{(n+1)}=\mathcal{D}_{\mu} \chi^{(n)}, n \geq 0
$$

Let us choose $\chi^{(0)}=0$. Then the first current

$$
J_{\mu}^{(1)}=\mathcal{D}_{\mu} \chi^{(0)}=\mathcal{A}_{\mu}
$$

This current is conserved by the equations of motion for $\mathcal{A}_{\mu}$, eq.(20). Now we would like to show that the $(n+1)^{t h}$ current constructed by the ansatz eq.(22) is conserved and the proof proceeds as follows:

$$
\partial^{\mu} J_{\mu}^{(n+1)}=\partial^{\mu} \mathcal{D}_{\mu} \chi^{(n)}=\mathcal{D}_{\mu} \partial^{\mu} \chi^{(n)}
$$

The last equality holds due to the fact that $\left[\partial^{\mu}, \mathcal{D}_{\mu}\right]=0$ as a consequence of the equation of motion eq.(20). Note, however that, $\partial^{\mu} \chi^{(n)}=\epsilon^{\mu \nu} J_{\nu}^{(n)}$ from eq.(22) and we can use the relation

$$
J_{\nu}^{(n)}=\mathcal{D}_{\nu} \chi^{(n-1)}, n \geq 1
$$

in eq. (23) to arrive at

$$
\partial^{\mu} J_{\mu}^{(n+1)}=\epsilon^{\mu \nu} \mathcal{D}_{\mu} \mathcal{D}_{\nu} \chi^{(n-1)}
$$


The right hand side of the equation vanishes since $\mathcal{A}_{\mu}$ is curvatureless. Thus we have shown that the current $J_{\mu}^{(n+1)}$ is conserved and we can construct an infinite sequence of conserved nonlocal currents through this procedure.

There are conserved charges $Q^{(n)}$ associated with each current and the charge is the space integral of the time component of the current

$$
Q^{(n)}=\int_{-\infty}^{+\infty} d x J_{0}^{(n)}(x, t)
$$

For example,

$$
Q^{(1)}=\int_{-\infty}^{+\infty} d x \mathcal{A}_{0}
$$

whereas the second charge has the form

$$
Q^{(2)}=\int_{-\infty}^{+\infty} d x \mathcal{D}_{0} \chi^{(1)}
$$

since $J_{0}^{(2)}$ can be related to $\chi^{(1)}$ through eq.(23). Thus,

$$
Q^{(2)}=-\int_{-\infty}^{+\infty} d x J_{1}^{(1)}(x, t)+\int_{-\infty}^{+\infty} d x J_{0}^{(1)}(x, t) \int_{-\infty}^{x} d x^{\prime} J_{0}^{(1)}\left(x^{\prime}, t\right)
$$

The first term in $(31)$ arises from the relation $\partial_{0} \chi^{(1)}=-J_{1}^{(1)}$ and the second term comes from the product $\mathcal{A}_{0} \chi^{(1)}$ and $\chi^{(1)}$ can be written as an integral over $J_{0}^{(1)}$ as is evident from eq.(22). We can compute all other conserved charges in following this prescription.

Thus far we have constructed the NCC in terms of $W_{A i}$ which belongs to $O(d, d)$. However, the fields appearing in the string effective action correspond to special form of $V$ given by eq.(10). Thus the relevant $W$ takes the following form 


$$
W=\frac{1}{2}\left(\begin{array}{cc}
E^{-1}+E-E^{-1} B & E-E^{-1} B \\
E-E^{-1}+E^{-1} B & E+E^{-1}+E^{-1} B
\end{array}\right)
$$

Therefore, all these currents can be ultimately expressed in terms of the moduli $G$ and $B$.

There are several comments in order at this stage. We have demonstrated that the two dimensional reduced string theory admits an infinite sequence of conserved nonlocal currents. In the case of the heterotic string where one includes the $U(1)^{16}$ Abelian gauge fields in the ten dimensional effective action the the scalars parametrise the coset $\frac{O(8,24)}{O(8) \otimes O(24)}$ for two dimensional spacetime. It obvious that the technique adopted here can be extended to construct the currents for the moduli of the heterotic string as well. The antisymmetric tensor field does not contribute to the two dimensional action. However, we made an assumption that the dilaton takes a constant value which simplified our calculations to some extent. If the dilaton carries spacetime dependence, how does it affect our results ? We note that for nonconstant dilaton the factor $e^{-\phi}$ will appear in front of the kinetic energy term of the M-matrix. We can redefine the M-matrix as was done by Bakas when he studied the effective two dimensional action. However, on this occasion, conditions given by eq.(20) and (21) cannot be satisfied simultaneously for a single vector potential constructed from $\mathrm{W}$ field. These two relations were crucial in this work to construct the set of NCC. Therefore, the present technique will not be useful to construct such currents. On the other hand, past experiences with supersymmetric nonlinear $\sigma$-models show that it might be still possible to obtain an infinite sequence of such currents. In the case of supersymmetric $\sigma$-model the vector that is curvatureless is not conserved and the one which is conserved is not curvatureless; nevertheless, it is possible to obtain a Lax pair and construct an infinite set of nonlocal conserved charges [16]. Another important issue is whether these classical conservation laws are valid in the quantised theory since anomalies might creep in when we 
define the currents with an appropriate normal ordering prescriptions. We hope to report our results on some of these problems in future.

To conclude, we have demonstrated the existence of an infinite set of nonlocal conserved current for the action under consideration.

Acknowledgments: I am grateful to John H. Schwarz and Ashoke Sen for very valuable discussions at the initial stage of this work. It is a pleasure to thank Alok Kumar for discussions and for carefully reading the manuscript. 


\section{References:}

[1] K. Kikkawa and M. Yamasaki, Phys. Lett. 149B (1984) 357; N. Sakai and I. Senda, Prog. Theor. Phys. 75 (1986) 692; T. Busher, Phys. Lett. 194B (1987) 59; Phys. Lett. 201B (1988) 466; Phys. Lett. 159B (1985) 127; V. Nair, A. Shapere, A. Strominger, and F. Wilczek, Nucl. Phys. B287 (1987) 402; A. Giveon, E. Rabinovici, and G. Veneziano, Nucl. Phys. B322 (1989) 167; M. J. Duff, Nucl. Phys. B335 (1990) 610, A. A. Tseylin and C. Vafa, Nucl. Phys. B372 (1992) 443; A. A. Tseytlin, Class. Quan. Gravity 9 (1992) 979.

[2] For recent reviews and detailed references see A. Sen Int. J. Mod. Phys. A9 (1994) 3707; A. Giveon, M. Porrati and E. Ravinovici, Phys. Rep.,C244 1994) 77.

[3] K. A. Meissner and G. Veneziano, Phys. Lett. 267B (1991) 33; G. Veneziano, Phys. Lett. 265B (1991) 287, K. A. Meissner and G. Veneziano, Mod. Phys. Lett. A6 (1991) 3397.

[4] M. Gasperini, J. Maharana and G. Veneziano, Phys. Lett. 272B (1991) 167; M. Gasperini. J. Maharana and G. Veneziano, Phys. Lett. 296 (1992) 51.

[5] A. Sen, Phys. Lett. 271B (1991) 295; A. Sen, Phys. Lett. 274B (1991) 34; S. F. Hassan and A. Sen, Nucl. Phys. B375 (1992) 103; S. P. Khastgir and A. Kumar, Mod. Phys. Lett. bf A6 (1991) 3365, S. Kar, S. P. Khastgir and A. Kumar, Mod. Phys. Lett. A7 (1992) 1545; J. Maharana, Phys. Lett. 296B (1992) 65.

[6] J. Maharana and J. H. Schwarz, Nucl. Phys. B390 (1993) 3. For earlier work see S. Ferrara, C. Kounnas and M. Porrati, Phys. Lett. B181 (1986) 263.

[7] J. Maharana Preprint Isaac Newton Institute for Mathematical Sciences, Cambridge NI94023, October 1994, (Phys. Lett.B in press).

[8] A. Font, L. Ibanez and F. Quevedo, Phys. Lett. 249B (1990) 35; S.-J. Rey, Phys. Rev. 
D43 (1991) 526; A. Sen Nucl. Phys. B404 (1993) 109; Phys. Lett. 303B (1993) 22; Mod.Phys. Lett. A8 (1993) 2023; J. H. Schwarz, Caltech Preprint CALT-68-1815; J. H. Schwarz and A. Sen Phys. Lett. 329B (1994) 105, A. Sen, Phys. Lett. 329B (1994) 217 ; J. H. Schwarz and A. Sen Nucl. Phys. B411 (1994) 35.

[9] A. Sen preprint TIFR-TH-94-19.

[10] G. Segal ( to appear).

[11] E. Witten (unpublished).

[12] I. Bakas Nucl. Phys. B428 (1994) 374.

[13] K. S. Narain Phys. Lett. bf 169B (1986) 41; K. S. Narain, H. Sarmadi and E. Witten Nucl. Phys. bf B279 (1987) 369. These works considered toroidal compactification with spacetime independent moduli and showed that they parametrise the coset $\frac{O(d, d)}{O(d) \otimes O(d)}$.

[14] M. Luscher and K. Pohlmeyer, Nucl. Phys. B137 (1978) 46; E. Brezin, C Itzykson, J. Zinn-Justin, J. B. Zubber, Phys. Lett. 82B (1979) 442; H. J. de Vega, Phys. Lett. 87B (1979) 233; A. T. Ogielski, Phys. Rev. D21 (1980) 3462; E. Witten, Phys. Rev. D16 (1978) 2991; P. di Vecchia and S. Ferrara, Nucl. Phys. B130 (1977) 93; E. Cremmer and J. Scherk, Phys. Lett. 74B (1978) 341; B. Zumino, Phys. Lett. 87B (1979) 203; E. Corrigan and C. K. Zachos, Phys. Lett. 88B (1979) 273; T. Curtright, Phys. Lett. bf 88B (1979) 276; Y. Y. Goldschmidt and E. Witten Phys. Lett. 91B (1980) 392; T. Curtright and C. K. Zachos, Phys. Rev. D21 (1980) 411; H. Eichenherr and M. Forger, Nucl. Phys. B156 (1979) 381; A. J. Mcfarlane, Phys. Lett. 82B (1979) 239; M. Dubois-Violette and Y. Georgelin, Phys. Lett. 82B (1979) 251.

[15] J. Maharana, Phys. Lett. 128B (1983) 411.

[16] J. Maharana, Lett. Math. Phys. 8 (1984) 284; J. Maharana, Ann. Inst. Henri Poincare bf45 (1986) 231 